\title{
From Gene to Drug: A Proof of Concept for a Plausible Computational Pathway
}

\author{
Sandhya Shenoy, Surojit Bose, Pankaj \\ Jayaram, B, Latha, N, Sharma, Poonam \\ Pooja Narang, Tarun Singhal, Gandhimathi, \\ Jain, Kumkum Bhushan, A., Praveen Agrawal, \\ Saher Afshan Shaikh, Vidhu Pandey, Samrat \\ Dutta, Gurvisha Sandhu, \\ Anuj Gupta, Shashank \\ Shekhar and Shailesh \\ Tripathi \\ Department of Chemistry \& \\ Supercomputing Facility for Bioinformatics \& Computational Biology, \\ Indian Institute of Technology, Hauz Khas, New Delhi-110016, India.
}

\begin{abstract}
The last decade has witnessed an exponential growth of sequence information in the field of biological macromolecules such as proteins and nucleic acids and their interactions with other molecules. Computational analyses for structure-function predictions based on such information are increasingly becoming an essential and integral part of modern biology. With rapid advances in the area, there is a growing need to develop efficient versatile bioinformatics software packages, which are hypothesis driven. 'Gene to Drug' is an attempt in this pursuit and an integration of heterologous applications of different technologies developed in-house and their translation into in silico products that cater to a majority of bioinformatics applications. This paper briefly presents the science behind Gene to Drug and how grid services and high performance computing platforms can be harnessed to bridge the gap between biomolecular sequence, structure and function.
\end{abstract}

\section{Introduction}

The world wide genome sequencing efforts and the concurrent developments in scientific software implementations on massively parallel computer architectures grant us the opportunity to dream that all genes and proteins in a cell would be identified, their spatial and temporal connections understood, their functions established and their structures determined so that drug design could be undertaken against suitable targets to develop individualized medicine almost in an automated way (Genome $\rightarrow$ Gene $\rightarrow$ Protein $\rightarrow$ Drug).
Currently without the help of any database, it is difficult to ascertain whether a given DNA sequence is a gene, and if it is a gene, what is the likely three-dimensional structure of its protein product. Also, the present drug design softwares fall short of expectations even if the structures of drug targets (proteins/DNA) are known.

Pursuing the dream of creating new science and converting it into technology and products useful to society, we have been developing a wide array of "Gene to Drug" software tools (www.scfbio-iitd.res.in), the major contributions being ChemGenome for genome analysis, Bhageerath for protein structure prediction and Sanjeevini for DNA/protein directed drug design.

\section{Softwares Developed}

\subsection{ChemGenome}

Suite A Novel Genome Analysis Software
iitd.res.in/chemgenome2)

Based on DNA energetics, a physicochemical model has been developed for whole genome analysis to identify protein coding regions in a genome. Accuracy as verified on 331 prokaryotic genomes exceeds $90 \%$ with a gene prediction rate of $95 \%$ in 206 genomes. Also, relatively high accuracy is observed in precise gene prediction (i.e. both start and stop site identification) where experimentally validated reliable data sets are available. In addition, analysis of all the 16 chromosomes of S.cerevisiae, a eukaryote, shows the generality of 
the methodology without the need for genome dependent database training.

The physiochemical properties of DNA considered in ChemGenome [1, 2] namely hydrogen bonding energy and stacking energy together with the rule of conjugates [3] seem to embed sufficient information for gene identification. The physicochemical model employs a three-dimensional (3-D) vector to represent double-helical deoxyribonucleic acid (DNA) base sequences, with each dimension denoting one facet of DNA recognition by proteins. Each of the 64 trinucleotides is assigned three coordinates, $x, y, z$, in the interval of -1 to $+1 \quad(x, y, z €[-1,+1]$, corresponding to the three proposed chemical properties of DNA. For a given DNA sequence (genome segment), the resultant vector is found by accumulating the $x, y$, and $z$ components of the individual codons $\left(X=\sum x, Y=\sum y, Z=\sum z\right)$. The orientation of this resultant vector from the origin is given by the direction cosines. Results on 331 genomes indicate that the vectors for genes and nongenes segregate quite nicely and are separated by a universal plane passing through the unit sphere [1], (Fig. 1). The relatively high sensitivity of the

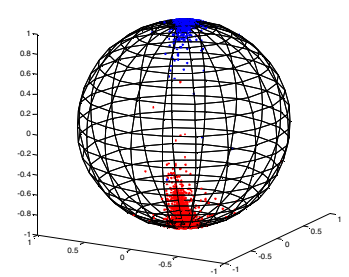

Figure 1a. Distribution of gene (north pole) and nongene (south pole) direction vectors for genome of E.coli (NC_000913), (sensitivity - 0.99; specificity 0.98 ; correlation coefficient -0.97 ).

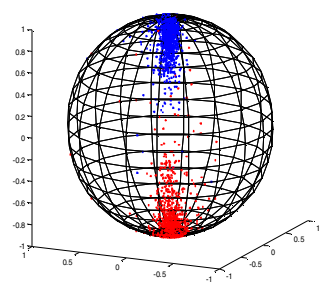

Figure 1b. Distribution of gene (north pole) and nongene (south pole) direction vectors for genome of B. subtilis ( $\mathrm{NC}_{-}$000964), (sensitivity - 1.00; specificity -0.84 ; correlation coefficient -0.82 ). method, coupled with the database independent and hypothesis driven nature of the algorithm should make it a useful tool for studies in structural genomics.

\subsection{Bhageerath}

A Protein Structure Prediction Software Suite (http://www.scfbioiitd.res.in/bhageerath/index.jsp )

Combining bioinformatics tools and $a b$ initio methodologies, considerable progress has been made towards a pathway that is computationally expeditious for tertiary structure prediction of small proteins. The software suite Bhageerath comprises eight modules configured to function independently or in a conduit. Starting with amino acid sequence and secondary structure information (helix/sheet/loop) of a protein in the first module, multiple three dimensional atomic level structures [4-6] are generated sampling the conformational space of the loop dihedrals in the second; in the third module a set of biophysical filters (persistence length, radius of gyration etc.) are applied which are designed to screen the trial structures to reduce the sample size. The resultant structures are refined in the fourth module by a Monte Carlo sampling in dihedral space to remove steric clashes / overlaps in 3-D space. An atomic level energy optimization is carried out in the fifth module and the structures scored based on energy in the sixth. Module seven reduces the probable candidates based on the protein regularity index of the $\varphi$ and $\psi$ dihedral values and module eight further reduces the structures selected to 10 using topological equivalence criterion and accessible surface area. Thus millions of possible structures for a given protein sequence are brought down to 10 candidate structures with the possibility of bracketing the native in these 10 . Results on a few small globular helical proteins (Fig. 2) have shown that the native like folds in the root mean square deviation (RMSD) range of 3-5 $\AA$ are captured in the best 10 structures energy-wise in all the cases without exception. The "needle in a hay stack" problem is thus reduced to choosing the best candidate from among the 10 lowest energy structures. Comparison with existing bioinformatics tools suggests the performance of the present methodology to be satisfactory and useful particularly when the database is deficient in sequence homologues. 


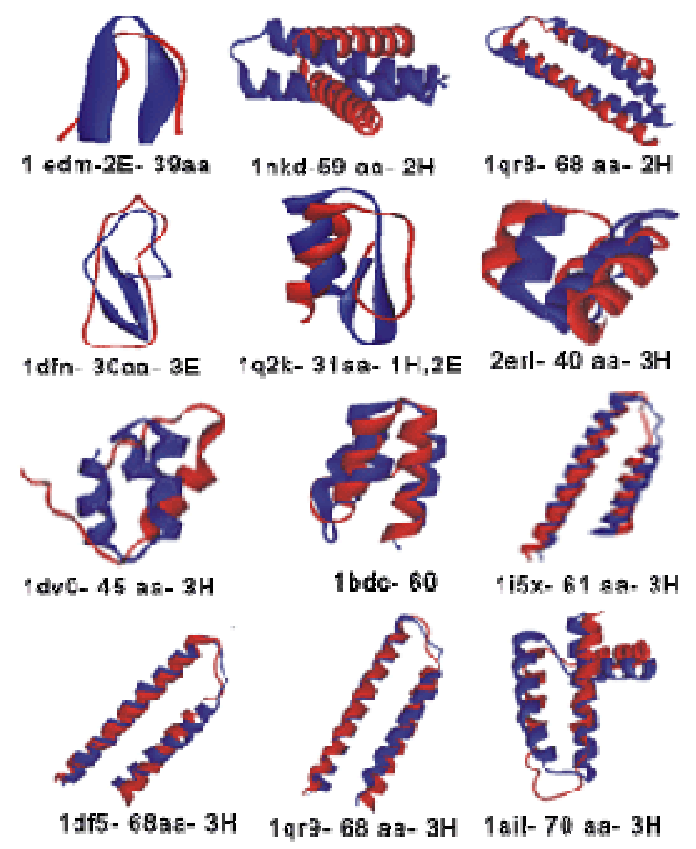

Figure 2. The lowest RMSD structure (red color) emerging from the Bhageerath pathway superimposed on the corresponding native (experimental/X-ray; blue color) structure for each of the globular test proteins. Results for other 38 systems are made available at the web site.

Preliminary work on alpha / beta systems has yielded encouraging results. Currently, the expected prediction time with Bhageerath web server for a 2-helix system (with one loop in between) is $\sim 4-5$ min while for a 3 helix system (with two loops in between), it is $\sim 2-3$ hours on a 32 processor cluster. Attempts to extend the methodology to larger systems with reduction in computational times are in progress.

\subsection{Sanjeevini}

Active-Site Directed Drug Lead Molecule Design Software Suite (http://www.scfbioiitd.res.in/research/drugdesign.htm )

A comprehensive software suite, Sanjeevini $[7,8]$ has been developed for target directed drug design. The software is system-independent and accomplishes lead-like molecule design based on binding affinities (the standard free energies of binding between target macromolecule and the candidate drug). The computational pathway paves way expressly towards lead molecule design starting with framing innumerable number of known or new candidate molecules out of a non-redundant small but versatile set of building blocks called templates. These candidates are then screened for oral bioavailability, followed by geometry optimization, determination of partial atomic charges and assignment of other force field parameters. The candidates thus prepared, are then docked in the active site of a given biological target using a Monte Carlo method and the interaction/binding energy is estimated using an all-atom energy based function. This binding affinity information is employed to judge whether the candidate molecule is leadlike. In propitious cases, molecular dynamics simulations can be performed with explicit solvent and salt on the biomolecular target, the candidate and the complex followed by a rigorous analysis of the binding free energy for further optimization. The methodology integrates tools of computational chemistry and molecular biophysics, some known and some new, to generate candidate molecules to any given target and to screen them based on binding affinities. The protocols developed draw strength from first principles and assure rigor, transferability and potential for automation. A proof of concept of the methodology developed obtained with the estrogen receptor target is represented in Fig. 3.

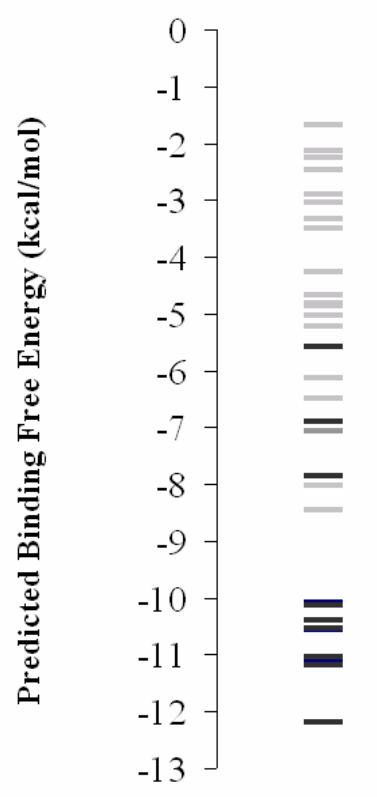

Figure 3. A typical separation achieved by Sanjeevini methodology of known marketed drugs from nondrugs for a biological target (Estrogen receptor in this case). 
As a part of Sanjeevini pathway, to enable a rapid scanning of candidate molecules, empirical energy based scoring functions have been developed for both, protein-drug [9] as well as DNA-drug [10-11] complexes. The scoring function for protein-drug complexes shows a high correlation (coefficient $\sim 0.92$ ) with experimental binding affinity data on 161 protein-drug complexes comprising 55 unique drug targets without the need for system dependent parameterization. The energy function for DNA-drug complexes shows correlation coefficients of 0.95 and 0.97 against experimental data on binding free energies and changes in melting temperatures upon DNAdrug binding for 50 DNA-drug complexes. These scoring function protocols have been web enabled for free usage by the scientific community.

\section{Products Developed}

\subsection{ChemGenome}

ChemGenome can be accessed at www.scfbio-iitd.res.in/chemgenome2. The web server offers platform-independent advantages to the user, requires no installation and presents a friendly browser interface for data entry and display. Users can input the whole genome sequence or part of genome of an organism. The sequence can be uploaded or alternatively pasted or typed into the query window of the browser. A tabular output displays the strand name, and the predicted gene boundaries.

\subsection{Bhageerath}

\footnotetext{
Bhageerath is a fully automated web enabled (http://www.scfbioiitd.res.in/bhageerath/index.jsp) $a b$ initio protein structure prediction software suite that is made available through a convenient user interface which returns 10 candidate structures for a given protein query sequence. A click on the Bhageerath server opens into a window wherein a user can paste a query protein sequence in FASTA format. The current version supports continuous sequences up to 100 amino acids. The user is prompted for amino acid range as secondary structural input. Upon submission the user receives a unique job id for his/her sequence. User has an option to provide an email ID to
}

receive an output link, which contains the results. The expected prediction time with Bhageerath web server depends on the length of the sequence, number of secondary structure elements and the number of structures accepted by biophysical filters for processing the energetics of each trial structure at the atomic level. It is currently able to process around 4-5 normally sized jobs per day on a 32 processor cluster.

\subsection{Sanjeevini}

Sanjeevini (http://www.scfbioiitd.res.in/research/drugdesign.htm) is a comprehensive active site-directed lead molecule design protocol. The scoring protocols associated with Sanjeevini for ranking candidate molecules for any biomolecular target are web-enabled for free access by the scientific community. These include BAPPL sever (http://www.scfbioiitd.res.in/software/drugdesign/bappl.jsp) that computes the binding free energy of a proteinligand complex, PreDDICTA (http://www.scfbio-

iitd.res.in/software/drugdesign/preddicta.jsp) which calculates the Drug-DNA interaction energy and Lipinski Filter (http://www.scfbioiitd.res.in/utility/LipinskiFilters.jsp) that checks whether a candidate satisfies Lipinski's rules.

\section{Conclusions and Perspectives}

"Gene to Drug" is the culmination of a functional prototype software suite completely based on ab initio methodologies, developed inhouse. ChemGenome is a novel gene identification model based on physicochemical properties of the double helix. The relatively high sensitivity of the method, coupled with database independent and hypothesis driven nature of the algorithm should make it a useful tool for studies in structural genomics. Bhageerath is energy based computational web server for tertiary structure predictions of proteins. The validation of the computational protocol on a few globular proteins shows that that the web server predicts a structure within an RMSD of 3-5 $\AA$ with respect to the native in the 10 lowest energy structures. Sanjeevini is a comprehensive drug design software suite, which could sort out drugs from non-drugs in an automated mode based on binding affinity calculations. The key driving forces for current 


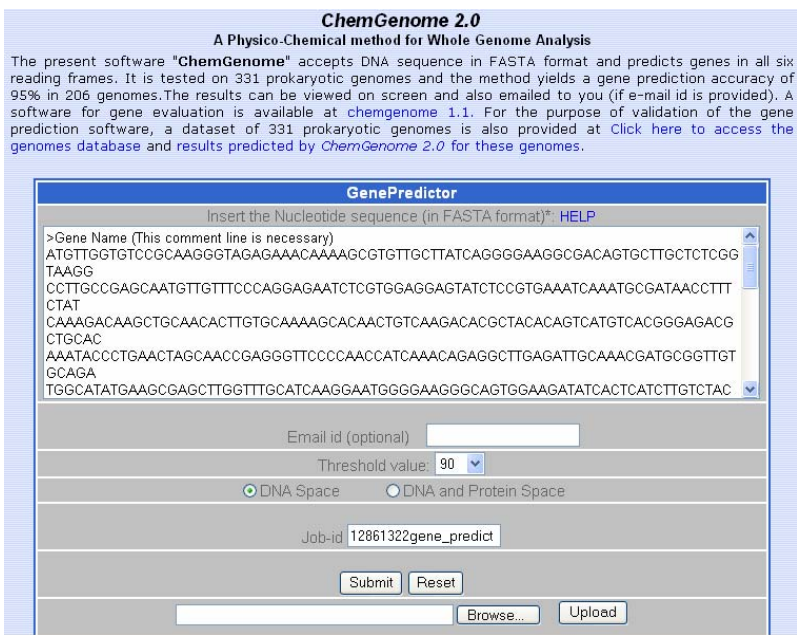

Figure 4. Snap shot of the front end of the ChemGenome software server

(http://www.scfbio-iitd.res.in/chemgenome/chemgenomenew.jsp)

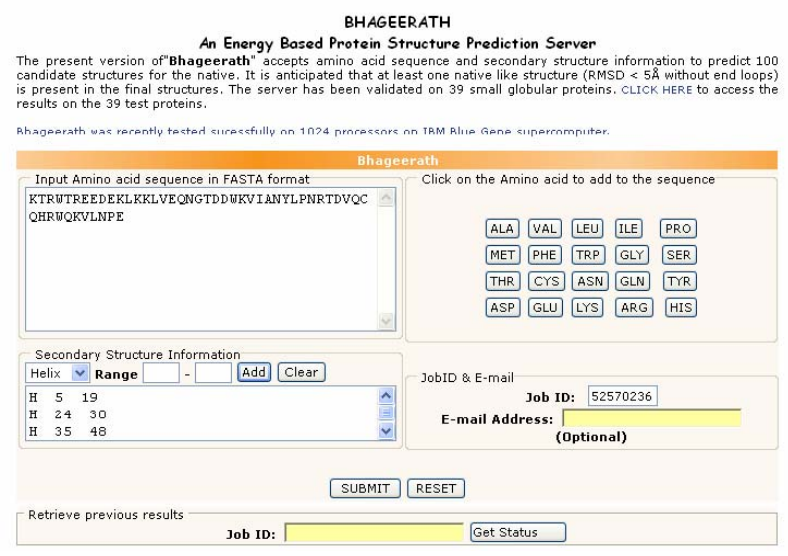

Figure 5. Snap shot of the front end of the Bhageerath software server

(http://www.scfbio-iitd.res.in/bhageerath/index.jsp)
PreDDICTA

Predict DNA-Drug Interaction strength by Computing $\Delta T \mathrm{~T}$ and Affinity of binding.

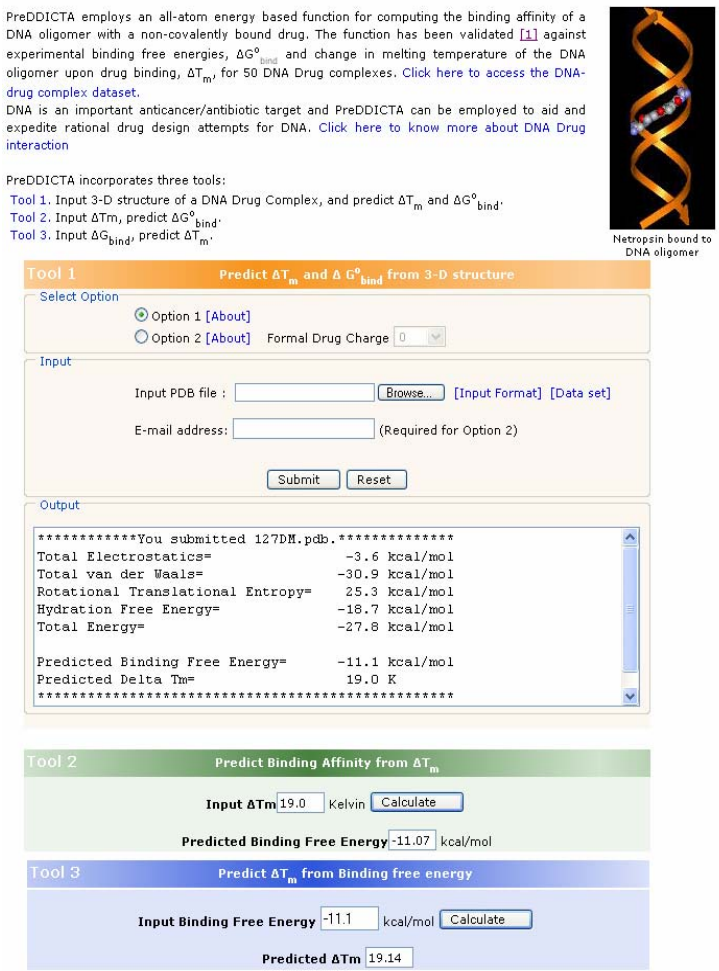

Figure 6. Snap shot of the front end of PreDDICTA server

(http://www.scfbio-iitd.res.in/software/drugdesign/ preddicta.jsp)

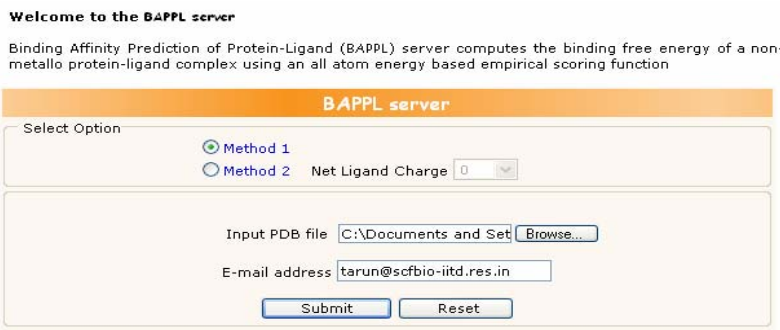

Figure 7. Snap shot of the front end of BAPPL server ( http://www.scfbioiitd.res.in/software/drugdesign/bappl.jsp)

The dream is to realize 'Gene to Drug' as a comprehensive bioinformatics application in a completely stand-alone automated pipeline and this appears feasible in the foreseeable future. 


\section{References}

1. S. Dutta, P. Singhal, P. Agrawal, R. Tomer, Kritee, E. Khurana, B. Jayaram, A Physico-Chemical Model for Analyzing DNA sequences, 2006, J. Chem. Inf. Mod., 46(1), 78-85.

2. P. Singhal, S. Dutta, B. Jayaram, A novel whole genome analysis software suite based on DNA energetics, 2006, Manuscript Communicated (chemgenome2).

3. B. Jayaram, Beyond the wobble: the rule of conjugates, 1997, J. Mol. Evol. 45, 704-705.

4. P. Narang, K. Bhushan, S. Bose, B. Jayaram, Protein structure evaluation using all atom energy based empirical scoring function, 2006, J. Biomol. Struct. Dyn. 23, 385-4006.

5. P. Narang, K. Bhushan, S. Bose, B. Jayaram, A computational pathway for bracketing native-like structures for small alpha helical globular proteins, 2005, Phys. Chem. Chem. Phys., 7, 2364.

6. B. Jayaram, Kumkum Bhushan, Sandhya R. Shenoy, Surojit Bose, Praveen Agrawal, Debashish Sahu and Vidhu S. Pandey, Bhageerath: An energy based web enabled computer software suite for limiting the search space of tertiary structures of small globular proteins, 2006, Manuscript Communicated.

7. N. Latha, T. Jain, P. Sharma, B. Jayaram, A free energy based computational pathway from chemical templates to lead compounds: a case study of COX-2 inhibitors, 2004, J. Biomol. Struct. Dyn, 21, 791-804.

8. N. Latha and B. Jayaram, A Binding Affinity Based Computational Pathway for Active-Site Directed Lead Molecule Design: Some Promises and Perspectives. 2005, Drug Design Reviews-Online, 2(2), 145.

9. T. Jain and B. Jayaram, All atom energy based computational protocol for predicting binding affinities of protein-ligand complexes. 2005, FEBS Letters, 579, 6659.

10. S. A. Shaikh, S. R. Ahmed and B. Jayaram, A Molecular Thermodynamic view of DNA-drug Interaction: A Case Study of 25 Minor Groove Binders.2004, Arch. Biochem. Biophys, 429, 81-99.

11. S. A. Shaikh and B. Jayaram, A Swift All-atom energy based Computational Protocol to Predict DNA-Drug Binding Affinity and delta Tm, 2006, Manuscript Communicated.

12. J. Besemer and M. Borodovsky, GeneMark: web software for gene finding in prokaryotes, eukaryotes and viruses. 2005, Nucleic Acids Research, 33, W451-W454.

13. L-H. Hung, S-C. Ngan, T. Liu and R. Samudrala, PROTINFO: new algorithms for enhanced protein structure predictions. 2005, Nucleic Acids Research, 33, W77-W80.

14. W. J. Jorgensen, The many roles of computation in drug discovery. 2004, Science, 303, 1813-1818. 